\title{
Breaking the Rectangle Bound Barrier against Formula Size Lower Bounds
}

\author{
Kenya Ueno \\ The Young Researcher Development Center and \\ Graduate School of Informatics, \\ Kyoto University \\ kenya@kuis.kyoto-u.ac.jp
}

\begin{abstract}
Karchmer, Kushilevitz and Nisan formulated the formula size problem as an integer programming problem called the rectangle bound and introduced a technique called the LP bound, which gives a formula size lower bound by showing a feasible solution of the dual problem of its LP-relaxation. As extensions of the LP bound, we introduce novel general techniques proving formula size lower bounds, named a quasi-additive bound and the Sherali-Adams bound. While the SheraliAdams bound is potentially strong enough to give a lower bound matching to the rectangle bound, we prove that the quasi-additive bound can surpass the rectangle bound.
\end{abstract}

\section{Introduction}

Proving formula size lower bounds is a fundamental problem in complexity theory as a weaker version of the circuit size lower bound problem and $\mathbf{P} \neq \mathbf{N P}$. A super-polynomial formula size lower bound for a function in NP implies $\mathbf{N C}^{1} \neq \mathbf{N P}$ [19]. As generalizations of the classical result of Khrapchenko [8] who proved an $n^{2}$ formula size lower bound for the parity function, there are a lot of techniques studied to improve formula size lower bounds. With all the efforts, improvements are far and few between. Karchmer, Kushilevitz and Nisan [6] formulated the formula size problem as an integer programming problem called the rectangle bound and introduced a technique called the LP bound, which gives a lower bound by showing a feasible solution of the dual problem of its LP-relaxation. At the same time, they also showed that it cannot prove a lower bound larger than $4 n^{2}$ for non-monotone formula size in general. Lee [14] proved that the LP bound [6] subsumes the quantum adversary bound of Laplante, Lee and Szegedy [10], which in turn subsumes most of known techniques such as Khrapchenko [8], its extension by Koutsoupias [9] and a key lemma used in the proof of Håstad [3] showing the current best formula size lower bound $n^{3-o(1)}$. Ueno [20] devised a stronger version of the LP bound by a cutting plane approach utilizing the theory of stable set polytope. However, it is difficult to determine the complete facet structure of the polytope associated with the formula size problem. Thus, improvements of the approach are limited. 
Lift-and-project methods systematically incorporate tighter and tighter constraints into any LP formulation. There are several lift-and-project methods such as Sherali and Adams [16,17], Balas, Ceria and Cornuejols [2] and Lovász and Schrijver [15] and Lasserre [11]. Laurent [12] gave a comparison among these techniques. Among the several techniques, the technique of Sherali and Adams [16, 17] has some advantages as the strongest one for LP formulations with relatively simpler descriptions. These techniques have attracted much attention from several contexts. In Section 5, we devise further another stronger version of the LP bound using the lift and project method of Sherali and Adams [16,17] and its application to the set partition polytope by Sherali and Lee [18]. It yields a convex hull of integral solutions and completely closes the integrality gap which causes the limit of the original technique. It is potentially strong enough to prove a lower bound matching to the rectangle bound.

More recently, Hrubeš, Jukna, Kulikov and Pudlák [5] discussed a notion of subadditive rectangle measures on combinatorial rectangles as a conceptual extension of a well-known notion of formal complexity measures. Inspired by this notion and the Sherali-Adams bound, we introduce yet another stronger version of the LP bound, which we name a quasi-additive bound, in Section 3. It directly gives a lower bound for formula size and the protocol partition number of the Karchmer-Wigderson game. In Section 4, we show that the quasi-additive bound can surpass the rectangle bound. So, the quasi-additive bound is not upperbounded by the rectangle bound in general. This is quite surprising because the quasi-additive bound can be seen as a simple extension of the LP bound of Karchmer, Kushilevitz and Nisan [6], which is originally defined as a relaxation of the rectangle bound. In fact, we can prove that the quasi-additive bound is potentially strong enough to prove the matching formula size lower bound for any Boolean function and the matching protocol partition number for any relation. Another interesting property of the quasi-additive bound is that we can derive a formula size lower bound for any Boolean function from a solution of the quasi-additive bound for the universal relation.

Since the Sherali-Adams and quasi-additive bounds are pure extensions of the LP bound, they can prove formula size lower bounds provable by any techniques subsumed by the LP bound. To extend a solution space for them, we introduce two useful techniques, named a cross argument and a triplet argument in Section 4 and Section 6, respectively. The cross argument is useful to break the rectangle bound barrier and applicable only for the quasi-additive bound. On the other hand, the triplet argument can cover a main part of techniques discussed in [20] and is applicable for both the Sherali-Adams and quasi-additive bounds. Breaking the rectangle bound barrier against formula size lower bounds implies a strong potential of the quasi-additive bound because the rectangle bound is not so far from formula size in general (See Theorem 2 and [13]) and almost all Boolean functions require formula size of at least $\Omega\left(2^{n} / \log n\right)$ (See, e.g., [21]). We hope that our generic techniques will be useful to surpass the best formula size lower bound $n^{3-o(1)}$ of Håstad [3]. 


\section{Preliminaries}

We assume that the readers are familiar with the basics of Boolean functions and linear programming. A Boolean function $f$ is called monotone if $x \leq y$ implies $f(x) \leq f(y)$ for all $x, y \in\{0,1\}^{n}$.

Definition 1 (Formula Size). A formula is a binary tree with each leaf labeled by a literal and each internal node labeled by either of the binary connectives $\wedge$ and $\vee$. A literal is either a variable or its negation. The size of a formula is its number of literals. We define formula size $L(f)$ of a Boolean function $f$ as the size of the smallest formula computing $f$. We also define $L_{m}(f)$ as the monotone formula size of a monotone Boolean function $f$ where a monotone formula is a formula without negations.

Karchmer and Wigderson [7] characterize formula size of any Boolean function in terms of a communication game. In the game, given a Boolean function $f$, Alice gets an input $x$ such that $f(x)=1$ and Bob gets an input $y$ such that $f(y)=0$. The goal of the game is to find an index $i$ such that $x_{i} \neq y_{i}$. Here, $x_{i}$ and $y_{i}$ denote the $i$-th bits of $x$ and $y$, respectively. The number of leaves in a best communication protocol for the Karchmer-Wigderson game is equal to the formula size of $f$. From the Karchmer-Wigderson game, we consider the following matrix called the communication matrix.

Definition 2 (Communication Matrix). Given a Boolean function $f$, its communication matrix is defined as a matrix whose rows and columns are indexed by $X=f^{-1}(1)$ and $Y=f^{-1}(0)$, respectively. Each cell of the matrix contains indices $i$ such that $x_{i} \neq y_{i}$. A combinatorial rectangle is a direct product $X^{\prime} \times$ $Y^{\prime}$ where $X^{\prime} \subseteq X$ and $Y^{\prime} \subseteq Y$. A combinatorial rectangle $X^{\prime} \times Y^{\prime}$ is called monochromatic if every cell $(x, y) \in X^{\prime} \times Y^{\prime}$ contains the same index $i$. To describe it simply, we define a relation $R_{f} \subseteq X \times Y \times\{1,2, \cdots, n\}$ as $R_{f}=$ $\left\{(x, y, i) \mid x \in X, y \in Y, x_{i} \neq y_{i}\right\}$. We can also define the monotone version of the communication matrix and the relation associated with a monotone Boolean function $f$ as $R_{f}^{m}=\left\{(x, y, i) \mid x \in X, y \in Y, x_{i}=1, y_{i}=0\right\}$.

To prove a lower bound, we sometimes restrict rows and columns of the communication matrix as $R^{\prime}=\left\{(x, y, i) \mid(x, y, i) \in R, x \in X^{\prime}, y \in Y^{\prime}\right\}$ for some $X^{\prime} \subseteq X$ and $Y^{\prime} \subseteq Y$. The number of leaves in a best communication protocol for the Karchmer-Wigderson game is equivalent to the following bound.

Definition 3 (Protocol Partition Number). For any combinatorial rectangle $X^{\prime} \times Y^{\prime}$, we call its partition a pair of $X_{1}^{\prime} \times Y^{\prime}$ and $X_{2}^{\prime} \times Y^{\prime}$ where $X^{\prime}=X_{1}^{\prime} \cup X_{2}^{\prime}$ and $X_{1}^{\prime} \cap X_{2}^{\prime}=\emptyset$, or a pair of $X^{\prime} \times Y_{1}^{\prime}$ and $X^{\prime} \times Y_{2}^{\prime}$ where $Y^{\prime}=Y_{1}^{\prime} \cup Y_{2}^{\prime}$ and $Y_{1}^{\prime} \cap Y_{2}^{\prime}=\emptyset$. The minimum number of disjoint monochromatic rectangles which recursively partition the communication matrix associated with a relation $R$ is defined as $C^{P}(R)$, called the protocol partition number.

Then, the theorem of Karchmer and Wigderson [7] can be stated as follows.

Theorem 1 ([7]). $\forall f, C^{P}\left(R_{f}\right)=L(f)$ and $C^{P}\left(R_{f}^{m}\right)=L_{m}(f)$. 
The minimum number of disjoint monochromatic rectangles which exactly cover all cells in the communication matrix gives a lower bound for the protocol partition number because a protocol partition itself is one of exact covers by disjoint monochromatic rectangles. We call it the rectangle bound defined as follows.

Definition 4 (Rectangle Bound). The minimum size of an exact cover by disjoint monochromatic rectangles for the communication matrix associated with a relation $R$ is defined as $C^{D}(R)$, called the rectangle bound.

On the relation between the protocol partition number and the rectangle bound, we know the following result. For the proof, we recommend [13].

Theorem 2 ([6]). $\forall R, C^{D}(R) \leq C^{P}(R) \leq 2^{O\left(\log ^{2} C^{D}(R)\right)}$.

Karchmer, Kushilevitz and Nisan [6] formulate the rectangle bound as an integer programming problem and give its LP relaxation.

Definition 5 (LP Bound). We define $\mathbf{L P}(R)$ as the optimal value of the following linear programming formulation associated with a relation $R$. Let $C$ be the set of all defined cells, $M$ be the set of all monochromatic rectangles and $Z_{r}$ be a variable associated with each monochromatic rectangle $r \in M$. Then, the $L P$-relaxation can be written as $\min \sum_{r \in M} Z_{r}$ such that $\sum_{r \ni c} Z_{r}=1$ for each cell $c \in C$ and $Z_{r} \geq 0$ for each $r \in M$. The dual problem can be written as $\max \sum_{c \in C} W_{c}$ such that $\sum_{c \in r} W_{c} \leq 1$ for each $r \in M$. Here, $W_{c}$ is a variable indexed by a cell $c \in C$.

From the duality theorem, showing a feasible solution of the dual problem gives a formula size lower bound.

Theorem 3 ([6]). $\forall f, \mathbf{L P}\left(R_{f}\right) \leq L(f)$ and $\mathbf{L P}\left(R_{f}^{m}\right) \leq L_{m}(f)$.

They define the universal relation to show the limitation of their technique.

Definition 6 (Universal Relation). The universal relation $U_{n}$ represents a matrix whose rows and columns are indexed by $X=Y=\{0,1\}^{n}$ and each cell $(x, y)$ is indexed by $\left\{i \mid x_{i} \neq y_{i}\right\}$. It is defined as $U_{n}=\left\{(x, y, i) \mid x \in\{0,1\}^{n}, y \in\right.$ $\left.\{0,1\}^{n}, x_{i} \neq y_{i}\right\} . C^{P}\left(U_{n}\right)$ and $C^{D}\left(U_{n}\right)$ are defined in the same way. Note that any cell $(x, y)$ where $x=y$ is undefined and are not counted for any partition. That is, monochromatic rectangles partition all defined cells without covering undefined cells.

It subsumes any relation $R_{f}$ as a submatrix. Its protocol partition number and rectangle bound also subsume those of any Boolean function as $C^{P}\left(R_{f}\right) \leq$ $C^{P}\left(U_{n}\right)$ and $C^{D}\left(R_{f}\right) \leq C^{D}\left(U_{n}\right)$ for any Boolean function $f$. They show a limitation of their technique by showing the following theorem.

Theorem 4 ([6]). $\forall f, \mathbf{L P}\left(R_{f}\right) \leq \mathbf{L P}\left(U_{n}\right) \leq 4 n^{2}$.

Thus, the LP bound and all the subsumed techniques [10] cannot prove a formula size lower bound larger than $4 n^{2}$. Limits inherent in previously known proof techniques which get stuck around $\Omega\left(n^{2}\right)$ heavily rely on the above theorem. 


\section{A Quasi-Additive Bound for Formula Size Lower Bounds}

In this section, we devise a stronger version of the LP bound, which is derived from a concept of subadditive rectangle measures by Hrubeš, Jukna, Kulikov and Pudlák [5] and inspired by the Sherali-Adams bound discussed in Section 5. We write $\Gamma$ as the set of combinatorial rectangles and $\Re$ as the set of real numbers. Hrubeš, Jukna, Kulikov and Pudlák [5] introduce a notion of rectangle measures. We call $\mu: \Gamma \mapsto \Re$ a subadditive rectangle measure if it satisfies the following two properties.

1. Normalization: $\mu(m) \leq 1$ for each monochromatic rectangle $m \in M$.

2. Subadditivity: $\mu(r) \leq \mu\left(r_{1}\right)+\mu\left(r_{2}\right)$ for each combinatorial rectangle $r \in \Gamma$ and its arbitrary partition into $r_{1}$ and $r_{2}$.

They show that $\mu(r)$ gives a lower bound for the protocol partition number $C^{P}(R)$ by a simple inductive argument and any relation $R$ where $r$ is the whole rectangle associated with the relation $R$. We can simply extend it for the universal relation as follows.

Lemma 1 ([5]). If $\mu$ is a subadditive rectangle measure for a relation $R$, then $\mu(X \times Y) \leq C^{P}(R)$, even in the case of the universal relation in which $\mu(r)=$ 0 for every combinatorial rectangle $r$ containing only an undefined cell, where $X \times Y$ is the whole matrix associated with the relation $R$.

A remarkable fact is that, if we strengthen the condition "Subadditivity" as

3. Additivity: $\mu(r)=\mu\left(r_{1}\right)+\mu\left(r_{2}\right)$ for each combinatorial rectangle $r \in \Gamma$ and its arbitrary partition into $r_{1}$ and $r_{2}$,

then it is equivalent to the dual problem of the original LP formulation of Karchmer, Kushilevitz and Nisan [6]. Then, we consider the following LP formulation.

Definition 7 (Quasi-Additive Bound). Let $C$ be the set of all cells, $M$ be the set of all monochromatic rectangles and $\Gamma$ be the set of all combinatorial rectangles associated with a relation $R$. We define $\mathbf{Q A}(R)$ as the optimal value of the following linear program formulation.

$$
\begin{aligned}
\max & \sum_{c \in C} V_{c} \\
\text { s.t. } & \sum_{c \in r} V_{c}+\sum_{c \notin r} V_{c, r} \leq 1, \quad(\text { for each } r \in M) \\
& \sum_{c \notin r_{1}} V_{c, r_{1}}+\sum_{c \notin r_{2}} V_{c, r_{2}} \geq \sum_{c \notin r} V_{c, r} . \\
& \left.\quad \text { for each } r \in \Gamma \text { and its arbitrary partition into } r_{1} \text { and } r_{2}\right)
\end{aligned}
$$

When we consider the universal relation, we fix $V_{c}=0$ for every undefined cell and $V_{c, r}=0$ for every combinatorial rectangle $r$ which only contains an undefined cell. 
We refer to it as the quasi-additive bound. It is stronger than the LP bound and gives a lower bound for the protocol partition number.

Lemma 2. $\forall R, \mathbf{L P}(R) \leq \mathbf{Q A}(R) \leq C^{P}(R)$.

Proof. If we set $V_{c, r}=0$ for each $c$ and $r$ of the quasi-additive bound, it is equivalent to the original LP bound. So, we have $\mathbf{L P}(R) \leq \mathbf{Q A}(R)$. To see $\mathbf{Q A}(R) \leq C^{P}(R)$, we regard $\mu(r)=\sum_{c \in r} V_{c}+\sum_{c \notin r} V_{c, r}$ as a rectangle measure. Then, we have $\mu(X \times Y)=\sum_{c \in C} V_{c}$, which is equal to the objective value of the quasi-additive bound, because $C=X \times Y$ is the whole rectangle associated with $R$. From the additivity of $V_{c}$, the conditions "Normalization" and "Subadditivity" is equivalent to the first and second constraints of the quasi-additive bound, respectively. Thus, if assignments of $V_{c}$ and $V_{c, r}$ satisfy all of the first and second constraints of the quasi-additive bound, $\mu(r)$ is a subadditive rectangle measure. Consequently, we have $\mathbf{Q A}(R) \leq C^{P}(R)$.

We can derive a formula size lower bound for any Boolean function from a solution for $\mathbf{Q A}\left(U_{n}\right)$ by calculating $\sum_{c \in r} V_{c}+\sum_{c \notin r} V_{c, r}$ where $r$ is $f^{-1}(1) \times f^{-1}(0)$. We can eliminate the redundancy of the quasi-additive bound by summarizing variables as $\bar{V}_{r}=\sum_{c \notin r} V_{c, r}$ for each combinatorial rectangle $r$ and adding a constraint $\bar{V}_{X \times Y}=0$. However, as we will show, this redundancy is useful to construct a solution for the quasi-additive bound. We can also prove that it is potentially strong enough to give the matching formula size lower bounds.

Theorem 5. $\forall R$, QA $(R)=C^{P}(R)$.

Proof. From the information of the protocol partition number $P(r)$ for each combinatorial rectangle $r$, we can construct a feasible solution whose objective value is equal to $P(X \times Y)\left(=C^{P}(R)\right)$. More precisely, we assign $V_{c}$ so as to satisfy $\sum_{c \in C} V_{c}=P(X \times Y)$. Then, we assign $V_{c, r}$ so as to satisfy $\sum_{c \notin r} V_{c, r}=$ $P(r)-\sum_{c \in r} V_{c}$. These assignments satisfy all the constraints of the quasi-additive bound and give the matching lower bound.

Corollary 1. $\forall f, \mathbf{Q A}\left(R_{f}\right)=L(f)$ and $\mathbf{Q A}\left(R_{f}^{m}\right)=L_{m}(f)$.

\section{A Cross Argument for the Quasi-Additive Bound}

In this section, we give an example of a relation for which the quasi-additive bound can surpass the rectangle bound. For this purpose, we devise a novel technique named a cross argument to give a solution of the quasi-additive bound.

Theorem 6. $\exists R, \mathbf{Q A}(R)>C^{D}(R)$.

Proof. We take 2 disjoint subsets of $\{0,1\}^{8}$ as

$$
\begin{aligned}
& X=\{10011000,00101001,00010110,01100100\} \\
& Y=\{01011101,10110101,01111010,10101110\}
\end{aligned}
$$




\begin{tabular}{|c||c|c|c|c|}
\hline & 01011101 & 10110101 & 01111010 & 10101110 \\
\hline \hline 10011000 & 1 & 5 & 1 & 4 \\
\hline 00101001 & 3 & 5 & 8 & 8 \\
\hline 00010110 & 7 & 7 & 6 & 4 \\
\hline 01100100 & 3 & 2 & 6 & 2 \\
\hline
\end{tabular}

Fig. 1. The Monotone Communication Matrix of the Relation $R$

\begin{tabular}{|c||c|c|c|c|}
\hline & 01011101 & 10110101 & 01111010 & 10101110 \\
\hline \hline 10011000 & a & b & c & d \\
\hline 00101001 & e & f & g & h \\
\hline 00010110 & h & g & f & e \\
\hline 01100100 & d & c & b & a \\
\hline
\end{tabular}

Fig. 2. 8 pairs of 2 cells from 16 cells

Then, we consider the monotone relation $R$ of $X$ and $Y$ as Figure 1. For the relation $R$, it is easy to see that $C^{P}(R) \leq 10, C^{D}(R) \leq 8$ by a cover with 8 maximal monochromatic rectangles and $\mathbf{L P}(R) \geq 8$ by assigning a weight $\frac{1}{2}$ for each cell.

Now, we prove $\mathbf{Q A}(R) \geq 10$. We assign $V_{c}=\frac{5}{8}$ for each cell. So, the total weight is 10 . To give an assignment rule of $V_{c, r}$, we consider 2 sorts of 8 pairs from 16 cells. One is composed of 8 pairs each of which has 2 cells with the same index in Figure 1. The other is composed of 8 pairs each of which has 2 cells with the same alphabet in Figure 2. For any 2 cells $c_{1}$ and $c_{2}$ and any combinatorial rectangle $r$ such that $c_{1} \notin r_{1}$ and $c_{2} \in r_{2}$, we define $\Delta_{c_{1}, r}\left(c_{2}\right)$ as follows. Let $c_{2}^{\prime}$ be the other cell which has the same index with $c_{2}$.

- If $c_{1}$ and $c_{2}$ have the same alphabet and $r$ contains $c_{2}^{\prime}$, we define $\Delta_{c_{1}, r}\left(c_{2}\right)=$ $-\frac{1}{8}$.

- If $c_{1}$ and $c_{2}$ have the same alphabet and $r$ does not contain $c_{2}^{\prime}$, we define $\Delta_{c_{1}, r}\left(c_{2}\right)=\frac{3}{8}$

- If $c_{1}$ and $c_{2}$ have different alphabets, we define $\Delta_{c_{1}, r}\left(c_{2}\right)=0$.

Then, we assign $V_{c_{1}, r}=\sum_{c_{2} \in r} \Delta_{c_{1}, r}\left(c_{2}\right)$ for any $c_{1} \notin r$. To verify the first constraints of the quasi-additive bound, it is sufficient to consider the 2 cases as monochromatic rectangles with either 1 or 2 cells. In both cases, we have $\sum_{c \in r} V_{c}+\sum_{c \notin r} V_{c, r}=1$.

From now on, we consider the second constraint $\sum_{c \notin r_{1}} V_{c, r_{1}}+\sum_{c \notin r_{2}} V_{c, r_{2}} \geq$ $\sum_{c \notin r} V_{c, r}$ where $r_{1}$ and $r_{2}$ be an arbitrary partition of a combinatorial rectangle $r$. We can assume that there is a pair of 2 cells $c_{1}$ and $c_{2}$ with the same alphabet in $r$. Otherwise, the constraint cannot be violated because $\Delta_{c_{1}, r}\left(c_{2}\right)=\Delta_{c_{1}, r_{1}}\left(c_{2}\right)=$ $\Delta_{c_{1}, r_{2}}\left(c_{2}\right)=0$ for any $c_{1}, c_{2} \in r$. We can also assume that the pair is partitioned into the 2 combinatorial rectangles as $c_{1} \in r_{1}$ and $c_{2} \in r_{2}$ in the following argument. We consider the case in which any pair of 2 cells $c_{1}$ and $c_{2}$ in $r$ with 
the same alphabet are also in $r_{1}$. In this case, the assignments of $\Delta$ concerned with any pair of $c_{1}$ and $c_{2}$ in $r$ do not decrease by the partition because $\frac{3}{8} \geq-\frac{1}{8}$. Moreover, no (negative) assignments of $\Delta$ do not appear by the partition. Hence, the constraint cannot be violated. The same thing is true for the case of $r_{2}$ instead of $r_{1}$.

Then, we define the diagonal pair $p^{d}$ of a pair $p$ in Figure 2 as the pair such that the 4 cells in $p^{d}$ and $p$ compose a $4 \times 4$ combinatorial rectangle. As an example, we consider the case when $c_{1}=[1, a] \in r_{1}$ and $c_{2}=[2, a] \in r_{2}$ without loss of generality. Here, we identify each cell by its index and alphabet in the figures. In this case, $r_{1}$ and $r_{2}$ must also partition the diagonal pair $c_{3}=[3, d]$ and $c_{4}=[4, d]$. Then, we take $c_{1}^{\prime}=[1, c], c_{2}^{\prime}=[2, c], c_{3}^{\prime}=[3, e]$ and $c_{4}^{\prime}=[4, e]$ be the 4 cells which have the same index with $c_{1}, c_{2}, c_{3}$ and $c_{4}$, respectively. If at least one of 4 pairs $\left(c_{1}, c_{1}^{\prime}\right),\left(c_{2}, c_{2}^{\prime}\right),\left(c_{3}, c_{3}^{\prime}\right)$ and $\left(c_{4}, c_{4}^{\prime}\right)$ has been already divided at the time of the partition into $r_{1}$ and $r_{2}$, then the changes of assignments of $\Delta$ concerned with only $c_{1}, c_{2}, c_{3}$ and $c_{4}$ are represented as either $\frac{3}{8}-\frac{1}{8}-\frac{1}{8}-\frac{1}{8} \geq 0$, $\frac{3}{8}+\frac{3}{8}-\frac{1}{8}-\frac{1}{8} \geq 0, \frac{3}{8}+\frac{3}{8}+\frac{3}{8}-\frac{1}{8} \geq 0$ or $\frac{3}{8}+\frac{3}{8}+\frac{3}{8}+\frac{3}{8} \geq 0$. Any of these does not cause a violation of the constraint. The same thing is true for any other pairs with the same alphabet. So, we can assume either $c_{1}, c_{1}^{\prime}, c_{3}, c_{3}^{\prime} \in r_{1}$ and $c_{2}, c_{2}^{\prime}, c_{4}, c_{4}^{\prime} \in r_{2}$, or $c_{1}, c_{1}^{\prime}, c_{4}, c_{4}^{\prime} \in r_{1}$ and $c_{2}, c_{2}^{\prime}, c_{3}, c_{3}^{\prime} \in r_{2}$. In both cases, we have $\sum_{c \notin r_{1}} V_{c, r_{1}}=0$ and $\sum_{c \notin r_{2}} V_{c, r_{2}}=0$ where $r_{1}$ and $r_{2}$ are $2 \times 4$ combinatorial rectangles. Since $r$ is the whole rectangle, we also have $\sum_{c \notin r} V_{c, r}=0$. Consequently, all the constraints of the quasi-additive bound are satisfied.

We also know a smaller relation $R^{\prime}$ of a $3 \times 3$ matrix having the gap between the rectangle bound and the protocol partition number. In this case, the gap is also smaller as $C^{P}\left(R^{\prime}\right)=6$ and $C^{D}\left(R^{\prime}\right)=5$.

The communication matrix discussed in the above proof is monotone and restricted. We can give an example of a non-monotone and whole communication matrix with the gap between the rectangle bound and the protocol partition number. Laplante, Lee and Szegedy [10] defined a 4-bit Boolean function $f_{A}$ called Ambainis' function following a similar construction of Ambainis [1]. (See [10] and [4] for more detailed treatment of Ambainis' function.) It outputs 1 when $x_{1} \leq x_{2} \leq x_{3} \leq x_{4}$ or $x_{1} \geq x_{2} \geq x_{3} \geq x_{4}$. In Figure 3 , we write the whole communication matrix $R_{f_{A}}$ of Ambainis' function. We can prove $\mathbf{L P}\left(R_{f_{A}}\right) \geq 8$ by assigning a weight $\frac{1}{2}$ for each cell whose number of indices is 1 and a weight 0 otherwise. In Figure 4, we show an upper bound of its rectangle bound $C^{D}\left(R_{f_{A}}\right) \leq 8$. (Distinct numbers and numbers with apostrophe represent distinct monochromatic rectangles.) Thus, any rectangle bound based techniques cannot improve the LP bound. On the other hand, we know a smallest formula of size 10 for Ambainis' function as

$$
\left(\left(x_{1} \vee \neg x_{2}\right) \wedge\left(x_{2} \vee \neg x_{3}\right) \wedge \neg x_{4}\right) \vee\left(\left(\neg x_{1} \vee x_{2}\right) \wedge\left(\neg x_{2} \vee x_{3}\right) \wedge x_{4}\right)
$$

Thus, $L\left(f_{A}\right)=C^{P}\left(R_{f_{A}}\right) \leq 10$. To the best of our knowledge, the quasi-additive bound is the first generic technique which can prove the matching lower bound $L\left(f_{A}\right) \geq \mathbf{Q A}\left(R_{f_{A}}\right) \geq 10$. 


\begin{tabular}{|c||c|c|c|c|c|c|c|c|}
\hline & 0100 & 0010 & 1010 & 0110 & 1001 & 0101 & 1101 & 1011 \\
\hline \hline 0000 & 2 & 3 & 1,3 & 2,3 & 1,4 & 2,4 & $1,2,4$ & $1,3,4$ \\
\hline 1000 & 1,2 & 1,3 & 3 & $1,2,3$ & 4 & $1,2,4$ & 2,4 & 3,4 \\
\hline 1100 & 1 & $1,2,3$ & 2,3 & 1,3 & 2,4 & 1,4 & 4 & $2,3,4$ \\
\hline 1110 & 1,3 & 1,2 & 2 & 1 & $2,3,4$ & $1,3,4$ & 3,4 & 2,4 \\
\hline 0001 & 2,4 & 3,4 & $1,3,4$ & $2,3,4$ & 1 & 2 & 1,2 & 1,3 \\
\hline 0011 & $2,3,4$ & 4 & 1,4 & 2,4 & 1,3 & 2,3 & $1,2,3$ & 1 \\
\hline 0111 & 3,4 & 2,4 & $1,2,4$ & 4 & $1,2,3$ & 3 & 1,3 & 1,2 \\
\hline 1111 & $1,3,4$ & $1,2,4$ & 2,4 & 1,4 & 2,3 & 1,3 & 3 & 2 \\
\hline
\end{tabular}

Fig. 3. The Communication Matrix of Ambainis' function

\begin{tabular}{|c||c|c|c|c|c|c|c|c|}
\hline & 0100 & 0010 & 1010 & 0110 & 1001 & 0101 & 1101 & 1011 \\
\hline \hline 0000 & 2 & 3 & 3 & 2 & $1^{\prime}$ & 2 & 2 & $1^{\prime}$ \\
\hline 1000 & 1 & 3 & 3 & 1 & $44^{\prime}$ & $4^{\prime}$ & $4^{\prime}$ \\
\hline 1100 & 1 & 3 & 3 & 1 & $4^{\prime}$ & $4^{\prime}$ & $4^{\prime}$ & $4^{\prime}$ \\
\hline 1110 & 1 & 2 & 2 & 1 & $2^{\prime}$ & $3^{\prime}$ & $3^{\prime}$ & $2^{\prime}$ \\
\hline 0001 & 2 & 3 & 3 & 2 & $1^{\prime}$ & 2 & 2 & $1^{\prime}$ \\
\hline 0011 & 4 & 4 & 4 & 4 & $1^{\prime}$ & $3^{\prime}$ & $3^{\prime}$ & $1^{\prime}$ \\
\hline 0111 & 4 & 4 & 4 & 4 & $1^{\prime}$ & $3^{\prime}$ & $3^{\prime}$ & $1^{\prime}$ \\
\hline 1111 & 1 & 2 & 2 & 1 & $2^{\prime}$ & $3^{\prime}$ & $3^{\prime}$ & $2^{\prime}$ \\
\hline
\end{tabular}

Fig. 4. An Upper Bound 8 for the Rectangle Bound of Ambainis' function

\section{Applying Sherali-Adams' Method to the LP Bound}

In this section, we strengthen the technique of Karchmer, Kushilevitz and Nisan [6] by a lift and project technique of Sherali and Adams [16,17]. While it is upperbounded by the rectangle bound, it is worthwhile to introduce because it has a similar structure with the quasi-additive bound. So, a solution for the SheraliAdams bound may be useful to give a solution for the quasi-additive bound.

We write $P \sqsubseteq M$ when all monochromatic rectangles in $P \subseteq M$ are disjoint. We also write $r \perp P$ when a monochromatic rectangle $r$ does not intersect any monochromatic rectangle in $P$. Let $C$ be the set of all defined cells, $M$ be the set of all monochromatic rectangles and $Z_{P}$ be a variable associated with a set of disjoint monochromatic rectangles $P$ in $M$. Now, we apply the lift and project technique to the LP-relaxation of the rectangle bound. The primal problem is written as follows.

$$
\begin{array}{ll}
\min & \sum_{r \in M} Z_{\{r\}} \\
\text { s.t. } & \sum_{r: c \in r \perp P} Z_{P \cup\{r\}}=Z_{P},\left(\text { for each } c \in C \text { and each } P \sqsubseteq M \text { s.t. } c \notin \bigcup_{r \in P} r\right. \text { ) } \\
& \quad \text { (for each } P \sqsubseteq M) \\
& Z_{\emptyset} \geq 0, \\
&
\end{array}
$$


This is a simple modification of Sherali and Lee [18], which discuss the set partition polytope in general. The dual problem is written as follows.

$$
\begin{array}{ll}
\max & \sum_{c \in C} W_{c, \emptyset} \\
\text { s.t. } & \left.\sum_{c \in r} W_{c, \emptyset}-\sum_{c \notin r} W_{c,\{r\}} \leq 1, \quad \text { (for each } r \in M\right) \\
& \sum_{r \in P} \sum_{c \in r} W_{c, P \backslash\{r\}}-\sum_{r \in P} \sum_{c \notin r} W_{c, P} \leq 0 . \text { (for each } P \sqsubseteq M \text { s.t. }|P|>1 \text { ) }
\end{array}
$$

Note that $W_{c, P}$ is defined only when $P \sqsubseteq R$ and $c \notin \bigcup_{r \in P} r$.

From the theory of Sherali and Adams $[16,17]$ and Sherali and Lee [18], giving the optimal solution for this dual problem shows the rectangle bound of the corresponding relation.

Definition 8 (Sherali-Adams Bound). We define $\mathbf{S A}_{h}(R)$ as the optimal value of the $h$-th level of the Sherali-Adams relaxation associated with $R$, which corresponds to the restriction of $W_{c, P}=0$ where $|P|>h$.

If we restrict the Sherali-Adams bound to the first level as $\mathbf{S A}_{1}(R)$, we have the following simplification of the dual problem by replacing $W_{c, \emptyset}$ by $V_{c}$ and $-W_{c,\{r\}}$ by $V_{c, r}$.

$$
\begin{aligned}
\max & \sum_{c \in C} V_{c} \\
\text { s.t. } & \sum_{c \in r} V_{c}+\sum_{c \notin r} V_{c, r} \leq 1, \quad(\text { for each } r \in M) \\
& \sum_{c \in r_{1}} V_{c, r_{2}}+\sum_{c \in r_{2}} V_{c, r_{1}} \geq 0 .\left(\text { for each }\left\{r_{1}, r_{2}\right\} \sqsubseteq M\right)
\end{aligned}
$$

Giving a feasible solution of the above formulation shows a lower bound for the rectangle bound and hence formula size. Since $C^{D}(R)$ is upper-bounded by the number of cells, we have the following theorem.

Theorem 7 ([16-18]).

$$
\forall R, \mathbf{L P}(R)=\mathbf{S A}_{0}(R) \leq \mathbf{S A}_{1}(R) \leq \cdots \leq \mathbf{S A}_{|C|}(R)=C^{D}(R) .
$$

\section{A Triplet Argument for the Sherali-Adams and Quasi-Additive Bounds}

In this section, we introduce a general technique named a triplet argument for giving a solution for the Sherali-Adams bound and the quasi-additive bound. The technique is applicable for any relation. For the explanation, we look at the 3 -bit majority function. A majority function $\mathbf{M A} \mathbf{J}_{2 l+1}$ with $2 l+1$ input bits outputs 1 if the number of 1 's in the input bits is greater than or equal to $l+1$ and 0 otherwise. Note that we know $\mathbf{L P}\left(R_{\mathbf{M A J}_{3}}\right) \leq 4.5$. 
Proposition 1. $\mathbf{S A}_{1}\left(R_{\mathbf{M A J}_{3}}\right) \geq 5$ and $\mathbf{Q A}\left(R_{\mathbf{M A J}_{3}}\right) \geq 5$.

Proof. We consider a communication matrix of the 3-bit majority function whose rows and columns are restricted to minterms and maxterms, respectively. We consider a triplet $\left(c_{1}, c_{2}, c_{3}\right)$ composed of 3 cells. Here, in the case of the 3-bit majority function, we consider a triplet of 3 cells each of which has 3 indices.

We firstly assume $V_{c, r}=0$ for any $c$ and $r$ and change assignments of $V_{c, r}$ (for each triplet sequentially in general case) without violating the second constraints of the Sherali-Adams bound and the quasi-additive bound in the following way. If a combinatorial rectangle $r$ contains 2 cells of the triplet, e.g., $c_{2}$ and $c_{3}$, we increment $V_{c_{1}, r}$ by $-2 \delta$ for the remaining 1 cell. If a combinatorial rectangle $r$ contains 1 cell of the triplet, e.g., $c_{1}$, we increment $V_{c_{2}, r}$ and $V_{c_{3}, r}$ by $\delta$ for the remaining 2 cells. In the case of 3 -bit majority function, we set $\delta=\frac{1}{6}$ for the triplet. Then, it is easy to verify the changes of assignments do not violate a constraint $\sum_{c \notin r_{1}} V_{c, r_{1}}+\sum_{c \notin r_{2}} V_{c, r_{2}} \geq 0$ for any 2 disjoint combinatorial rectangles $r_{1}$ and $r_{2}$. We also have $\sum_{c \notin r}\left(V_{c, r}-V_{c, r_{1}}-V_{c, r_{2}}\right) \leq 0$ for any combinatorial rectangle $r$ and its arbitrary partition into $r_{1}$ and $r_{2}$. Thus, all the second constraints cannot be violated.

We give a weight $-\frac{1}{3}$ for each cell in the triplet and a weight 1 for each cell from the other 6 cells with 1 index. Then, we can also verify the assignments satisfies all the first constraints of the quasi-additive bound and the SheraliAdams bound. As a consequence, we have the lower bound of 5 .

Combining the triplet argument explained in the above proof and the idea of [20], we can prove the same lower bound $L\left(\mathbf{M A J}_{2 l+1}\right) \geq \frac{(l+1)^{2}}{1-\epsilon(l)}$ where $\epsilon(l)=$ $\frac{l^{2}(l+1)}{6 \cdot\left(\begin{array}{c}2+1 \\ l\end{array}\right)}$ as that of [20] for the majority function by both of the first level of the Sherali-Adams bound and the quasi-additive bound.

\section{Conclusions}

In this paper, we introduced the novel general techniques proving formula size lower bounds, the Sherali-Adams bound and the quasi-additive bound, as extensions of the LP bound of Karchmer, Kushilevitz and Nisan [6]. In particular, we proved that the quasi-additive bound can surpass the rectangle bound by the cross argument. We also showed that the quasi-additive and Sherali-Adams bounds can cover a main part of techniques discussed in the previous paper [20] by the triplet argument.

\section{Acknowledgment}

The author thanks Troy Lee for joyful discussions leading to the triplet argument and Kazuyuki Amano for sharing an efficient program computing the protocol partition number. This research is supported by the Kyoto University Hakubi Project and the Japan Society for the Promotion of Science. 


\section{References}

1. A. Ambainis. Polynomial degree vs. quantum query complexity. Journal of Computer and System Sciences, 72(2):220-238, 2006.

2. E. Balas, S. Ceria, and G. Cornuéjols. A lift-and-project cutting plane algorithm for mixed 0-1 programs. Mathematical Programming, 58:295-324, 1993.

3. J. Håstad. The shrinkage exponent of De Morgan formulas is 2. SIAM Journal on Computing, 27(1):48-64, Feb. 1998.

4. P. Høyer, T. Lee, and R. Špalek. Negative weights make adversaries stronger. In Proceedings of the 39th Annual ACM Symposium on Theory of Computing (STOC 2007), pages 526-535, 2007.

5. P. Hrubeš, S. Jukna, A. Kulikov, and P. Pudlák. On convex complexity measures. Theoretical Computer Science, 411:1842-1854, 2010.

6. M. Karchmer, E. Kushilevitz, and N. Nisan. Fractional covers and communication complexity. SIAM Journal on Discrete Mathematics, 8(1):76-92, Feb. 1995.

7. M. Karchmer and A. Wigderson. Monotone circuits for connectivity require superlogarithmic depth. SIAM Journal on Discrete Mathematics, 3(2):255-265, May 1990.

8. V. M. Khrapchenko. Complexity of the realization of a linear function in the case of $\pi$-circuits. Mathematical Notes, 9:21-23, 1971.

9. E. Koutsoupias. Improvements on Khrapchenko's theorem. Theoretical Computer Science, 116(2):399-403, Aug. 1993.

10. S. Laplante, T. Lee, and M. Szegedy. The quantum adversary method and classical formula size lower bounds. Computational Complexity, 15(2):163-196, 2006.

11. J. B. Lasserre. Global optimization with polynomials and the problem of moments. SIAM Journal on Optimization, 11(3):796-817, /2001.

12. M. Laurent. A comparison of the Sherali-Adams, Lovász-Schrijver and Lasserre relaxations for 0-1 programming. Mathematics of Operations Research, 28(3):470496, 2003.

13. T. Lee. Kolmogorov Complexity and Formula Size Lower Bounds. PhD thesis, University of Amsterdam, January 2006.

14. T. Lee. A new rank technique for formula size lower bounds. In Proceedings of the 24th Annual Symposium on Theoretical Aspects of Computer Science (STACS 2007), Lecture Notes in Computer Science 4393, pages 145-156. Springer, 2007.

15. L. Lovász and A. Schrijver. Cones of matrices and set-functions and 0-1 optimization. SIAM Journal on Optimization, 1(2):166-190, may 1991.

16. H. D. Sherali and W. P. Adams. A hierarchy of relaxation between the continuous and convex hull representations for zero-one programming problems. SIAM Journal on Discrete Mathematics, 3:411-430, 1990.

17. H. D. Sherali and W. P. Adams. A hierarchy of relaxations and convex hull characterizations for mixed-integer zero-one programming problems. Discrete Applied Mathematics, 52(1):83-106, 1994.

18. H. D. Sherali and Y. Lee. Tighter representations for set partitioning problems. Discrete Applied Mathematics, 68(1-2):153-167, 1996.

19. P. Spira. On time-hardware complexity tradeoffs for boolean functions. In Proceedings of the 4th Hawaii Symposium on System Sciences, pages 525-527, 1971.

20. K. Ueno. A stronger LP bound for formula size lower bounds via clique constraints. In Proceedings of the 26th Annual Symposium on Theoretical Aspects of Computer Science (STACS 2009), Leibniz International Proceedings in Informatics (LIPIcs) 3, pages 685-696. Schloss Dagstuhl-Leibniz-Zentrum fuer Informatik, 2009.

21. I. Wegner. The Complexity of Boolean Functions. Wiley-Teubner, 1987. 